\title{
Uncertainty in fibre strength characterisation due to uncertainty in measurement and sampling randomness
}

\author{
Sébastien Joannès • Faisal Islam • Lucien Laiarinandrasana
}

Received: 10 May 2019 / Accepted: 2 April 2020 / Published online: 5 May 2020

\begin{abstract}
Carbon fibres have exceptional mechanical properties and are used for critical structural applications such as composite pressure vessels and aerospace components. For such high performance applications, reliability-based designs and lifetime assessments require very accurate strength models. Accuracy of the predictions made by composite strength models depend on realistic material properties of constituents, which are used as input. In practice however, fibre strength properties reported by different sources show significant variations. The work described here aims at understanding the influence of measurement uncertainty and sampling randomness on the uncertainty in calculated tensile strength distribution parameters. Tensile strength data for T700 carbon fibres obtained from single fibre testing process has been analysed for uncertainties. A parametric bootstrap method has been used for the evaluation. It has been shown that although both the causes studied of uncertainty are critical, the sampling randomness has a larger influence on the uncertainty of fibre strength, as compared to the uncertainty due to measurement. Choosing an insufficient sample size for analysis can thus result in uncertain or even inaccurate fibre strength properties, which would limit the reliability of composite strength models. The knowledge of the causes and effects of these uncertainties can help in taking appropriate measures for improving the accuracy of results. This would thereby enhance the capability of composite strength models to estimate the behaviour of different composite structures more accurately.
\end{abstract}

Corresonding author: Sébastien Joannès

E-mail: sebastien.joannes@mines-paristech.fr

MINES ParisTech, PSL University, Centre des Matériaux (CMAT), CNRS UMR 7633, BP 8791003 Evry Cedex, France
Keywords Weibull distribution · Single fibre - Tensile tests · Confidence interval · Variability 


\section{Author notes 2020}

\section{Licensing}

Copyright (C) 2020. This manuscript version is made available under the CC-BY-NC-ND 4.0 license.

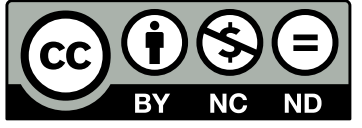

http://creativecommons.org/licenses/by-nc-nd/4.0

You are free to:

- Share - copy and redistribute the material in any medium or format as long as you follow the license terms.

Under the following terms:

- Attribution - You must give appropriate credit, provide a link to the license, and indicate if changes were made. You may do so in any reasonable manner, but not in any way that suggests the licensor endorses you or your use.

- NonCommercial - You may not use the material for commercial purposes.

- NoDerivatives - If you remix, transform, or build upon the material, you may not distribute the modified material.

\section{Citation}

Please use the following citation when referencing this work:

S. Joannès, F. Islam, L. Laiarinandrasana, Uncertainty in Fibre Strength Characterisation Due to Uncertainty in Measurement and Sampling Randomness, Applied Composite Materials 27 (3) (2020) 165-184. doi:10.1007/s10443-020-09803-9

\section{Correspondence}

Correspondence concerning this article should be addressed to: sebastien.joannes@mines-paristech.fr

or to the address mentioned at:

(D) https://orcid.org/0000-0002-9360-1643 


\section{Introduction}

Fibre reinforced composites are known to be light weight, have high specific strength and excellent weathering stabilities. These properties make them ideal to be used for very critical structural applications such as pressure vessels and components for aerospace applications [2, $3]$. These two applications represent about $40 \%$ of the composite market in volume [4]. In-service safety and reliability assessment are key challenges for these loadbearing applications and require great care to be taken during their design. Although using high design safety factors on the influential parameters make it possible to secure the structures in a deterministic manner, it conceals the extent of the risk that is being taken in doing so. Structural reliability analysis based on omnipresent uncertainties has the capability to provide the required risk information to engineers, if it could be appropriately conducted. To do that, the inherent variability in the properties of the constituent materials should be determined or predicted as best as possible $[5,6]$.

The design of composite material structures is assisted by computational models for predicting their mechanical properties at both component and structural level. Critical structures such as composite pressure vessels which are used for the storage of hydrogen or other gases at high pressures require very accurate computational models, so that the predictions can be used with confidence in industrial applications.

Previous studies of the research group have been focussed on developing computational composite strength models to predict the strength and damage behaviour of composite materials and structures leading to reliable lifetime assessment. Since fibres are the principal load bearing components in fibre reinforced composites, the effective mechanical and damage properties of composites are influenced mainly by the properties of fibres $[7,8]$. The accuracy of the predictions made by composite strength models would therefore be highly dependent on the accuracy of the input fibre strength distribution.

To assess the predictions made by composite strength and damage models, worldwide failure exercises have been conducted $[9,10]$. The predictions made by the different models analysed were found to spread over a large range, thus suggesting the reason for the lack of confidence in such models. A small change in input data can cause a significant variation in model predictions such as the failure strength or the time to failure of a composite structure and any error in input fibre strength properties would lead to inaccurate model predictions. This behaviour has been highlighted in one of the previous works [11], where the time to failure for composite structures has been shown to depend strongly on the uncertainty in input data. For example, the time to failure for the composite specimen studied increased by a factor of ten on decreasing the standard deviation of fibre strength distribution to a third of its initial value. Similarly, a scatter in the mechanical properties of actual composite specimens was observed experimentally in the same study. It is therefore required that fibre properties used as model input are determined with the best possible accuracy and any associated uncertainty in quantified appropriately.

Mechanical properties of fibres available in literature from different sources are usually not consistent, even for the same type of fibres. There is significant variability, especially in the tensile strength. This makes the selection of input data ambiguous and raises doubts on the reliability of the model predictions. In practice, the characterization of fibre properties is known to be very challenging. Their very small cross-sections, morphological variations, or simply the sampling randomness and sample size used for analysis, directly affect determination of fibre properties especially tensile strength. They make the characterization uncertain and may lead to significant variations in results $[12,13]$. This paper aims at understanding the causes of uncertainties in fibre strength characterization and their effect on the tensile strength distribution parameters. Accuracy of experimentally determined fibre strength is strongly dependent on measurement accuracy of the instruments used. No measurement is perfectly accurate and there is some degree of uncertainty associated with every result [14]. The uncertainty would be even larger for very brittle materials such as carbon fibre which is one of the most popular reinforcement for structural composites. Since fibre strengths are typically represented using statistical distributions, the distribution parameters are also affected by these inaccuracies. Apart from the limited precision of measurement tools used[15], uncertainties could also arise from many other sources such as the inconsistency of the individual performing the measurement, choice of sample size used, sampling error, etc. The representativity of the experimental fibre tensile strength data set that is used for determining a statistical distribution to characterize the tensile strength behaviour can be a major source of uncertainty in distribution parameters [16]. Since an estimate of uncertainty is essential to the proper interpretation of any experiment, it is important to understand the influence of these factors on the tensile strength results and the uncertainties that they introduce with them. The accuracy of determined fibre strength distribution could be significantly improved by minimising the effect of these factors. This would also enhance the capability of 
composite strength models to estimate the behaviour of different composite materials and structures more accurately.

\subsection{Fibre strength and Weibull distribution}

Fibre strength is typically represented by using statistical distribution functions. Classical descriptive statistics, describing the main features of a data set in quantitative terms such as the mean, the median or the standard deviation are not sufficient. Statistical inference, deducing properties of an underlying probability distribution, should be preferred. Weibull proposed a distribution that accounts for the intrinsic variation in strength of brittle fibres and could be used to represent their strength behaviour [17]. Some studies have proposed modifications to this distribution but the standard 2-parameter Weibull probability distribution function given by Equation 1 has been the most widely used representation $^{1}$. The present study also uses this function, whose scope is discussed in a forthcoming work to be published.

$P_{\mathrm{R}}(\sigma)=1-\exp \left[-\left(\frac{L}{L_{0}}\right)\left(\frac{\sigma}{\sigma_{0}}\right)^{m}\right]$

where, $P_{\mathrm{R}}(\sigma)$ is the probability of fibre failure for an applied stress level $\sigma, L$ being the characteristic gauge length, $L_{0}$ the reference gauge length, $\sigma_{0}$ the scale parameter, $m$ the shape parameter or Weibull modulus. The scale parameter, $\sigma_{0}$, represents the strength at which of the fibres would fail. The shape parameter, $m$, represents the scatter in strength around the mean value. Fibre strengths are typically reported in terms of their shape and scale parameters.

\subsection{Variability in Weibull parameters}

It has been observed that there have been differences in results reported by different authors for the same type of fibres. T700 carbon fibres are very popular in fibre reinforced composites for structural applications. The Weibull distribution parameters for the tensile strength of these type of fibres resulting from different studies is given in Table 1 . The scale parameter, $\sigma_{0}$, is dependent on the gauge length used, so all reported scale parameter values have also been normalised for a gauge length of $30 \mathrm{~mm}$ using the Weibull scaling equation ${ }^{2}$ given

\footnotetext{
1 In this equation, it is assumed that the section of the fibre remains constant along its length.

2 This extrapolation technique must be used with caution and various scientific papers address this complex topic [18, $19,12]$.
}

by Equation 2, derived from Equation 1. $\sigma_{0,1}$ and $\sigma_{0,2}$ are scale parameter values determined for fibre gauge lengths of $L_{0,1}$ and $L_{0,2}$, respectively and $m$ is the shape parameter of the obtained distribution.

$\sigma_{0,2}=\sigma_{0,1}\left(\frac{L_{0,1}}{L_{0,2}}\right)^{1 / m}$

The normalised scale parameter values are also given in Table 1. Fig. 1(a) shows the reported shape parameter values for different gauge lengths given by different authors. Fig. 1(b) shows the scale parameter values normalised for a gauge length of $30 \mathrm{~mm}$. Values determined by using the single fibre testing (SFT) methodology are depicted with a circle and the size of the circles are in proportion to the sample size that was used to determine these results, as also given in Table 1. Values determined by using the fragmentation and bundle testing methodologies are depicted by diamond and square shaped markers, respectively. The shape parameter is observed to vary between 3.5-17.5 which is a huge variation. The normalised scale parameter values are also observed to have a big variation and lie between 1.9-8.5 GPa.

The possible reasons for the differences in results need to be discussed in order to have a better understanding of the reliability that could be associated with each result available in literature. Authors usually do not comment on the uncertainty that can be associated with their results, or possible sources of errors which 63 (29 affect the accuracy of the determined results. It has only been mentioned by a few studies that there are possibilities of uncertainty in results, but the exact causes and effects have not been investigated in detail. The present study focusses on studying some possible sources of uncertainties in estimating the fibre strength distribution parameters.

Some possible causes for uncertainties in parameters of Weibull distribution are as follows:

(1) Testing method: Different authors have used different experimental techniques for the determination of Weibull parameters. These mainly include the single fibre testing process [20], fragmentation process [21] and the fibre bundle testing process [22]. Some other less commonly used methodologies have also been used [12]. The effect of experimental techniques on Weibull parameters have been discussed by Andersons et al. [23].

(2) Analysis method: To determine Weibull parameters from experimental results by fitting the data to a model, different data reduction techniques are used such as the least squares method, maximum likelihood method, etc. Different rank determining 
methods also exist. The chosen analysis method may have some influence on the obtained results.

(3) Measurement uncertainty: Another factor that could affect the results are measurement errors [6]. Measurements are made using instruments and in general, every measurement has imperfections which give rise to an error in the result. This may also contribute to the uncertainty in the final results [14].

(4) Sampling randomness: To determine perfectly accurate Weibull parameters, an infinite number of fibres would have to be tested. Since doing that is impracticable, there would always be some doubts on the representativeness of the chosen sample. This may be a major source of variability.

(5) Inconsistency in the material itself: Since fibre strength is controlled mainly by the distribution of defects, slight variations in manufacturing conditions could lead to differences in the microstructure of the material. This may also impact the properties of the fibres produced. Moreover, the fibre is often tested from a bobbin - as it is the case in this work but it can also be tested after a few transformation steps and this can also lead to differences.

Most of the time, none of these details are given in the publications, which makes the quantitative comparisons complicated.

The focus of the present study is on the effect of two causes mentioned in the above list: measurement errors (3) and sampling randomness (4). They have been discussed in this article in sections 2 and 3 , respectively.

\section{Uncertainty due to measurement}

Uncertainty in theory represents the state of unpredictability or unreliability. Uncertainty of the Weibull distribution parameters would mean the lack of knowledge or confidence on the values of the calculated quantities. Since the distribution parameters are calculated using experimentally measured fibre strength data, the uncertainties or errors in measured fibre strengths would also be transferred into the resulting Weibull distribution. Fibre strengths are calculated using measured quantities coming from instruments. Each measured quantity has some amount of measurement uncertainty or error. This results in an uncertainty or error in the calculated fibre strength as well [15].

Fibre strength data was generated by conducting single fibre tensile tests using T700 Carbon fibres while following the guidelines described in ASTM C1557 14 [32]. All tests were conducted using a universal tensile tester which was developed by Bunsell [33] and has been improved over the years with the introduc-

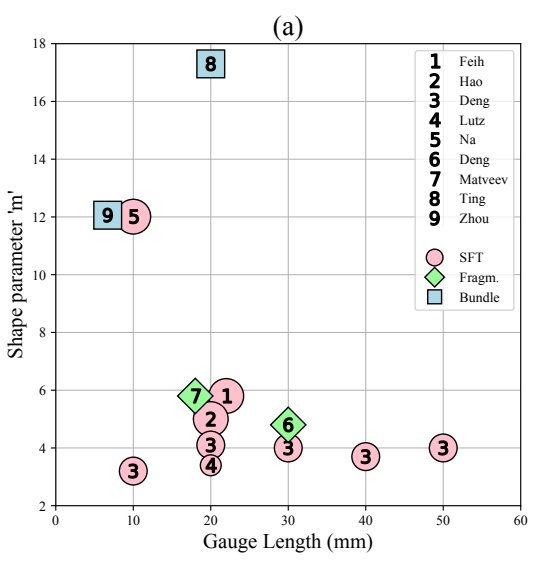

(b)

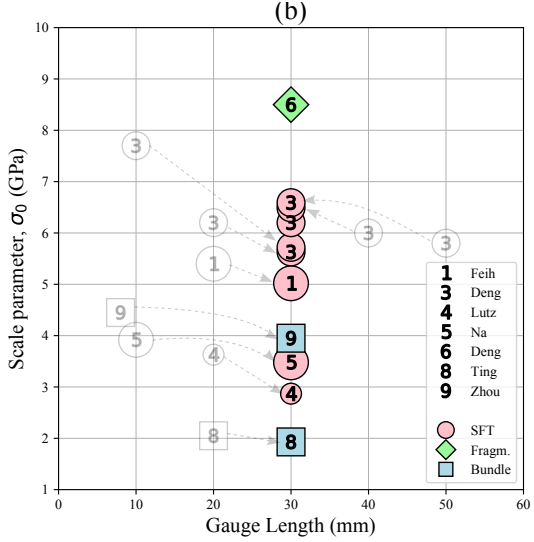

Fig. 1 Weibull parameter values for T700 carbon fibres reported in literature for: a) Shape parameter b) Scale parameter. Circular, diamond and square-shaped markers represent results from the single fibre tests (SFT), fibre fragmentation tests and fibre bundle tests, respectively. The scale parameter values have been normalised for a gauge length of $30 \mathrm{~mm}$ using the Weibull scaling equation (Equation 2). The values originally reported are also shown.

tion of more advanced transducers and sensors and a change from the vertical position to the horizontal position of the system. Those changes have for example made it possible to study the effect of temperature on fibre strength and this improved setup has been used very extensively to study many kinds of technical, natural and textile fibres $[34,35,36,37,38]$. Single fibres were extracted from the fibre bundles and mounted on paper frames containing cut-out slots of $30 \mathrm{~mm}$ in the middle which fixed the gauge length, $L_{0}$, as shown in Fig. 2. The diameter of each individual fibre specimen was measured at different locations along the fibre length before the fibre was subjected to the tensile test. A Mitutoyo laser scanning micrometre (LSM500) system was used for the measurements. This system allows rapid, non-contact, and accurate fibre dimensional measurements. The fibre is usually fixed between two grips 
Table 1 Weibull distribution parameters for T700 carbon fibre in literature (Scale parameter normalized for $L_{0}=30 \mathrm{~mm}$ )

\begin{tabular}{|c|c|c|c|c|c|c|c|c|}
\hline S.N. & $\begin{array}{l}\text { Type of } \\
\text { fibre }\end{array}$ & $\begin{array}{l}\text { Testing } \\
\text { method }\end{array}$ & $\begin{array}{l}\text { Gauge } \\
\text { length } \\
(\mathrm{mm})\end{array}$ & $\begin{array}{l}\text { Sample } \\
\text { size, N }\end{array}$ & Shape, $m$ & $\begin{array}{l}\text { Scale, } \sigma_{0} \\
(\mathrm{GPa})\end{array}$ & $\begin{array}{ll}\text { Scale, } & \sigma_{0} \\
\text { (Norm.) } & \\
(\mathrm{GPa}) & \end{array}$ & Author \\
\hline 1 & $\mathrm{~T} 700$ & SFT & 20 & 30 & 5.6 & 5.4 & 5.02 & Feih [24] \\
\hline 2 & T700 & SFT & 20 & 30 & 5.39 & - & - & Hao [25] \\
\hline \multirow{5}{*}{3} & \multirow{5}{*}{ T700 } & \multirow{5}{*}{ SFT } & 10 & 20 & 3.5 & 7.7 & 5.63 & \multirow{5}{*}{ Deng [26] } \\
\hline & & & 20 & 20 & 5 & 6.2 & 5.72 & \\
\hline & & & 30 & 20 & 4 & 6.2 & 6.20 & \\
\hline & & & 40 & 20 & 3.7 & 6 & 6.49 & \\
\hline & & & 50 & 20 & 4 & 5.8 & 6.59 & \\
\hline 4 & T700 & SFT & 20 & 10 & 4.68 & 3.63 & 2.87 & Lutz [27] \\
\hline 5 & T700 & SFT & 10 & 30 & 12.01 & 3.92 & 3.58 & $\mathrm{Na}[28]$ \\
\hline 6 & T700 & Fragm. & 30 & 6 & 4.8 & 8.5 & 8.50 & Deng [26] \\
\hline 7 & T700 & Fragm. & 20 & - & 5.6 & 5.47 & - & Matveev [29] \\
\hline 8 & T700 & Bundle & 20 & 15 & 17.53 & 1.98 & 1.93 & Ting [30] \\
\hline 9 & T700 & Bundle & 8 & - & 12.06 & 4.41 & 3.95 & Zhou [31] \\
\hline
\end{tabular}

and a perpendicular laser beam scans across the fibre. The time of obstruction of the light is recorded and the diameter is calculated. A total of 30 fibres were measured following the same procedure and tested as per the given standard. A constant displacement rate of 1 $\mathrm{mm} / \mathrm{min}$ was applied to stretch the fibres until failure. The failure load was recorded using a $150 \mathrm{~g}$ Sensotec Model 31 load-cell and the fibre extension was measured using an LVDT displacement transducer ACT1000A from RDP Electronics Ltd.

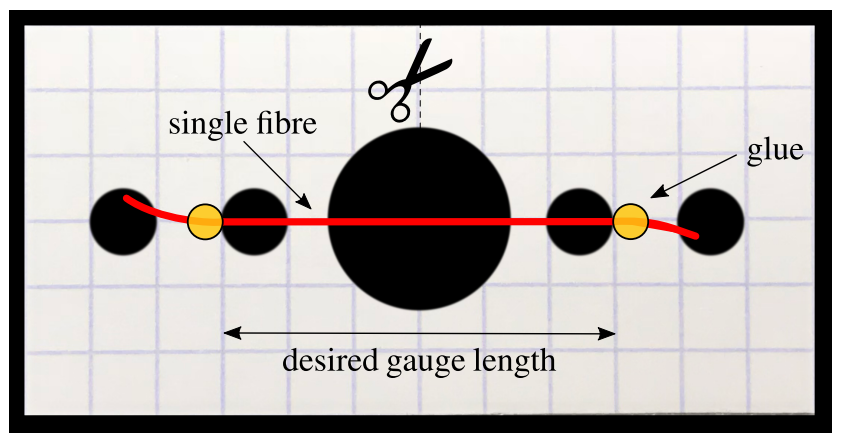

Fig. 2 The actual card frame used for preparing the fibre specimens.

The measurement uncertainty has been calculated using the law of propagation of uncertainty, and following the guidelines mentioned in the guide to the expression of uncertainty in masurement (GUM) [14]. The best estimates ${ }^{3}$ for the calculated fibre strengths $\sigma$ and the corresponding measurement uncertainty $u_{c}(\sigma)$

\footnotetext{
3 the value calculated analytically without considering any uncertainties.
}

for 30 fibres is given in Table 2, in increasing order of strength values (taken from [15]).

To determine the effect of measurement uncertainty on the uncertainty in fibre strength distribution, determination of the representative Weibull distribution and its parameters using fibre strength data is described in section 2.1 and estimation of the corresponding uncertainty on Weibull distribution parameters is described in section 2.2 .

\subsection{Fibre strength distribution}

Once the fibre tensile strength data set is generated, the next step is to represent the strength values using Weibull analysis. The statistical analysis of a series of tensile tests on single fibres can be carried out in the following way: The results of $n$ fibres are arranged in order of increasing failure stresses, i.e. $\sigma_{1}<\cdots<\sigma_{i}<$ $\cdots<\sigma_{n}$ and an experimental cumulative failure probability (or unreliability) $P_{\mathrm{R}}\left(\sigma_{i}\right)$, should be provided for each fibre of rank $i$ having failed at a stress $\sigma_{i}$. Unless an infinite number of fibres are tested, which is clearly impossible, the most general expression must take into account the possibility of fibres breaking at lower stresses than that of the weakest specimen as well as breaking at higher stresses than the strongest fibre tested. Rank methods determine the way an estimated unreliability is associated with each failure level. Various expressions of this probability have been proposed, depending mainly on the sample size. For sample sizes greater than 100 , the problem of small sample bias be- 
Table 2 Best estimates and measurement uncertainty of fibre strength for all tests from [15]

\begin{tabular}{ccc|ccc|ccc}
\hline S.N. & $\begin{array}{c}\sigma \\
(\mathrm{GPa})\end{array}$ & $\begin{array}{c}u_{c}(\sigma) \\
(\mathrm{GPa})\end{array}$ & S.N. & $\begin{array}{c}\sigma \\
(\mathrm{GPa})\end{array}$ & $\begin{array}{c}u_{c}(\sigma) \\
(\mathrm{GPa})\end{array}$ & S.N. & $\begin{array}{c}\sigma \\
(\mathrm{GPa})\end{array}$ & $\begin{array}{c}u_{c}(\sigma) \\
(\mathrm{GPa})\end{array}$ \\
\hline 1 & 1.69 & 0.07 & 11 & 3.23 & 0.10 & 21 & 4.36 & 0.08 \\
2 & 1.78 & 0.07 & 12 & 3.28 & 0.11 & 22 & 4.45 & 0.12 \\
3 & 1.97 & 0.06 & 13 & 3.48 & 0.08 & 23 & 4.49 & 0.05 \\
4 & 2.23 & 0.03 & 14 & 3.50 & 0.18 & 24 & 4.72 & 0.09 \\
5 & 2.56 & 0.06 & 15 & 3.52 & 0.17 & 25 & 4.73 & 0.12 \\
6 & 2.72 & 0.03 & 16 & 3.68 & 0.14 & 26 & 4.85 & 0.06 \\
7 & 2.73 & 0.05 & 17 & 3.75 & 0.09 & 27 & 5.44 & 0.31 \\
8 & 2.82 & 0.05 & 18 & 3.82 & 0.07 & 28 & 5.68 & 0.08 \\
9 & 2.93 & 0.06 & 19 & 4.04 & 0.06 & 29 & 5.99 & 0.17 \\
10 & 3.03 & 0.09 & 20 & 4.15 & 0.12 & 30 & 6.92 & 0.10 \\
\hline
\end{tabular}

come insignificant and the mean rank method could be used to estimate the unreliability using Equation 3.

$P_{\mathrm{R}}\left(\sigma_{i}\right)=\frac{i}{n+1}$

For smaller sample sizes, the median rank method is to be preferred over other methods since it provides positions at a specific confidence level (i.e. 50\%) and is thus best suited to some further work on confidence limits. The median rank method, estimates unreliability values based on the failure order number and the cumulative binomial distribution. If the sample size is sufficient, i.e. more than 50 fibres, Benard's approximation can be used, as given by Equation 4 .

$P_{\mathrm{R}}\left(\sigma_{i}\right)=\frac{i-0.3}{n+0.4}$

However, it is better to use median rank tables or to directly calculate the median ranks from the cumulative binomial distribution. The median rank is, at a $50 \%$ confidence level, the value that the true probability of failure has for the $i^{\text {th }}$ failure out of a sample of $n$ fibres. It is calculated by solving Equation 5 for $p$.

$0.5=\sum_{k=i}^{n}\left(\begin{array}{l}n \\ k\end{array}\right) p^{k}(1-p)^{(n-k)}$ where $\left(\begin{array}{l}n \\ k\end{array}\right)=\frac{n !}{k !(n-k) !}$

Following this method, the calculated fibre strength data from Table 2 is fitted to a 2-parameter Weibull distribution using the maximum likelihood estimation method and the corresponding Weibull plot is shown in Fig. 3. The points represent the experimental fibre strength values and the straight line is the best fitted 2-parameter Weibull distribution model obtained from the cumulative density function given by Equation 1 . The shape and scale parameters of the obtained Weibull distribution are $m=3.23$ and $\sigma_{0}=4.19 \mathrm{GPa}$, respectively. It is also given in Table 3 .

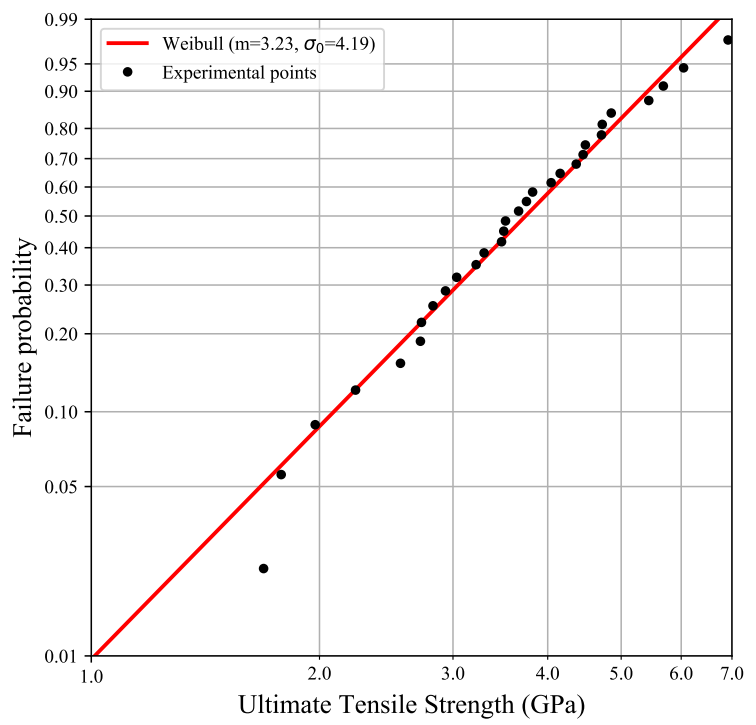

Fig. 3 Estimated Weibull plot for experimentally determined fibre strength

Table 3 Weibull parameters for experimentally determined fibre strengths

\begin{tabular}{cc}
\hline $\begin{array}{c}\text { Shape parameter } \\
m\end{array}$ & $\begin{array}{c}\text { Scale parameter } \\
\sigma_{0}(\mathrm{GPa})\end{array}$ \\
\hline 3.23 & 4.19 \\
\hline
\end{tabular}


Each of the calculated fibre strengths has some level of uncertainty associated with it due to the uncertainty of measurement. Having a measurement uncertainty practically means that if it was somehow possible to go back in time and conduct a tensile test using the same fibre again to determine the strength, the result obtained may have been different. It would deviate from the best estimate due to uncertainty in measurement of the quantities on which it depends. Consequently, the corresponding Weibull distribution for fibre strength would also be different, and would have different values of shape and scale parameters. This means that there would also be uncertainties associated with the calculated Weibull shape and scale parameters. The estimation of uncertainty in Weibull parameters has been discussed in the following section.

\subsection{Estimation of measurement uncertainty on Weibull parameters}

Since it is practically impossible to physically test fibres that have already been tested, uncertainty in Weibull parameters arising from measurement cannot be obtained from experimental results. Simulated values for fibre strength have thus been used to determine their Weibull distributions. Measurement uncertainty is usually assumed to follow a normal distribution [14]. A large number of fibre strength data sets similar to the one experimentally determined were simulated, under the assumption that uncertainty in fibre strength follows a normal distribution. Taking the experimentally calculated fibre strength results as reference, virtual fibre strength values were randomly generated from the assumed normal distribution with the experimental fibre strength value as the mean, and the calculated uncertainty as the standard deviation of the distribution, respectively. One hundred virtual fibre strength points were simulated around each experimental point, as also depicted in Fig. 4. Virtual fibre strength data sets were generated by randomly extracting one point from each of these clusters to form a data set of 30 virtual fibre strength data points. A total of 100 similar data sets were generated with each set comprising of 30 fibre strength values, same size as that of the reference data set. Each simulated data set was fitted to a Weibull distribution, all of which are plotted in Fig. 4. The shape and scale parameters for each distribution are determined and the results are summarized in Table 4 . The shape parameter $m$ was found to vary between 3.09 and 3.38 with an average value of 3.22 and having a standard deviation of 0.06 . The best estimate for $m$ and the uncertainty $\Delta m$ can be represented by the average value and the standard deviation, respectively. The scale parameter $\sigma_{0}$ was found to vary between $4.12 \mathrm{GPa}$ and $4.25 \mathrm{GPa}$ with an average value of $4.18 \mathrm{GPa}$ and having a standard deviation of $0.03 \mathrm{GPa}$. The shape and scale parameters in terms of best estimates and associated uncertainties are given as follows:

$$
\begin{gathered}
m=3.22 \text { with } u(m)=0.06 \\
\sigma_{0}=4.18 \mathrm{GPa} \text { with } u\left(\sigma_{0}\right)=0.03 \mathrm{GPa}
\end{gathered}
$$

The guide to uncertainty measurement recommends calculation of an expanded uncertainty $U(x)$ for a quantity of interest $x$, for commercial and industrial applications. The expanded uncertainty is calculated based on the required level of confidence using Equation 6, where $t_{95}$ is a coverage factor for a $95 \%$ level of confidence. When the number of degrees of freedom tends to $\infty$, the value of $t_{95}$ is 1.96 , and is determined by using the T-distribution table. A confidence level at $95 \%$ was chosen because of its wide use but for applications requiring more "confidence", higher levels can be chosen.

$U_{95}(x)=t_{95} \times u(x)$

The expanded uncertainties can be calculated following Equation 6 which gives $U_{95}(m)=0.12$ and $U_{95}\left(\sigma_{0}\right)=$ 0.06 . The shape and scale parameters in terms of their expanded uncertainties are given as follows:

$m=3.22 \pm 0.12$, for a confidence level of $95 \%$

$\sigma_{0}=4.18 \pm 0.06 \mathrm{GPa}$, for a confidence level of $95 \%$

Table 4 Weibull parameters for virtual fibre strength data sets

\begin{tabular}{ccc}
\hline & $\begin{array}{c}\text { Shape } \\
\text { parameter } \\
m\end{array}$ & $\begin{array}{c}\text { Scale } \\
\text { parameter } \\
\sigma_{0} \\
(\mathrm{GPa})\end{array}$ \\
\hline Mean & 3.22 & 4.18 \\
Standard deviation & 0.06 & 0.03 \\
Expanded uncertainty & 0.12 & 0.06 \\
\hline
\end{tabular}

The variation in shape parameter values is about $4 \%$ from the best estimate value on each side whilst the shape parameter is shown to vary by about $1.5 \%$ on each side from its best estimate. The variation in parameter values by itself is small. However, for modelling the strength of composite materials, Weibull parameters are required for very short gauge lengths, sometimes in the range of a few microns. The parameters 


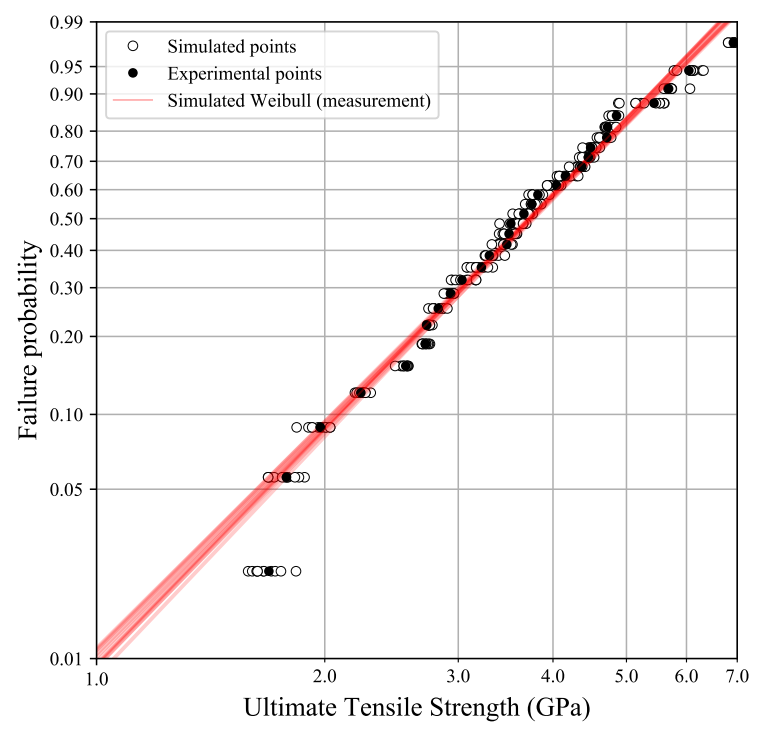

Fig. 4 Weibull distributions simulated by varying fibre strength data points within the uncertainty region

are usually determined at gauge lengths which are large enough to be experimentally feasible and the results are extrapolated to shorter gauge lengths using Equation 2. When Weibull parameters are extrapolated to shorter gauge lengths, the uncertainties associated with them may become very significant. If these values are used as input to model the properties of any product or structure which uses such fibres, there would be a significant uncertainty in those properties as well. Hence, it is very important that such uncertainties in fibre strength distribution parameters are clearly reported and also appropriately incorporated during computer strength modelling of any structure which uses such fibres.

From this analysis, it can be seen that the uncertainty resulting due to the uncertain measurement of individual fibre strength is not very substantial. However, this would be true only if the fibre strength data is generated using a sufficiently long gauge length. This is because the effect of measurement limitations is small for tests conducted at large gauge lengths and the measured tensile strength is more accurate. However, for shorter gauge lengths, measurement uncertainty of the instruments and experimental constraints limits the accuracy of the fibre strength data generated. Their effect on the uncertainty of the distribution parameters in this case would also be expected to be significant [15]. For minimizing the uncertainty due to measurement inaccuracies, it would thus be recommended that a sufficiently large gauge length is used for generating the fibre strength data.

\section{Uncertainty due to sampling randomness}

The determination of uncertainty in fibre strength and its Weibull distribution parameters described in section 2 considers only the effect of measurement. Individual measurement uncertainty for each input quantity is considered for obtaining the overall uncertainty on end results. However, uncertainties in Weibull distribution parameters could also arise due to many other factors such as the representativity of the chosen sample to determine the distribution, especially in the case when single fibre test results are used. Many challenges are associated with fibre characterization. Due to their delicate nature testing fibres is a time consuming and cumbersome process. Only a limited number of fibres can be tested to determine the Weibull distribution that could represent the fibre strengths for the whole fibre population in consideration. This limits the choice of sample size and conducting Weibull analysis using a fibre strength data set of an insufficient sample size may lead to sampling error. To comprehend the effect of sampling on the uncertainty of results, a confidence interval can be calculated, as described in the following section.

\subsection{Confidence Interval}

Statistics uses a random sample from a larger population and estimates results based on this sample. However, it is uncertain how well the results from the taken sample represent the underlying population. This uncertainty can be addressed by computing a confidence interval which provides a range of values which are likely to contain the results of the investigated parameter. Confidence intervals are calculated at a given confidence level, since the interval does not necessarily include the true value of the parameter. For example, a confidence interval calculated at a 95\% confidence level, also known as a 95\% confidence interval, has the following meaning: When a parameter is estimated many times from samples similar to the original one, then the real and unknown value of the parameter for the whole population will lie within the confidence interval, 95\% of the times [39]. The term "confidence interval" refers to the interval estimate along with its confidence coefficient.

A confidence interval is sometimes considered identical to a credible interval; the two are however different from each other. The first corresponds to a frequentist approach while the second to a Bayesian approach. A 95\% credible interval has the following meaning: Given an observed data, there is a $95 \%$ probability that the true value of an unknown parameter lies within 
a credible interval. The calculated numerical values for the two intervals may often be similar, but they have different meanings and should not be confused with each other. For the present study, a 95\% confidence interval was calculated for the parameters of the Weibull distribution. The steps involved in the calculation are described in Section 3.2.

\subsection{Calculation of confidence interval for Weibull parameters}

There are different methods of calculating a confidence interval for Weibull statistics, such as Fisher's matrix bounds, Beta-Binomial bounds, likelihood ratio bounds, Monte Carlo bounds, etc $[40,41]$. They are breifly described as follows:

Fisher's matrix bounds The Fisher's matrix bounds are parametric in nature, i.e. they follow an underlying distribution, with parameters. It has been found that they tend to be more optimistic than rank based nonparametric bounds, specially for small sample sizes. Thus, many statisticians prefer to use non-parametric bounds.

Beta Binomial bound The Beta Binomial bound follows the non-parametric approach, i.e. no underlying distribution is assumed, for calculating the confidence interval and is less mathematically intensive. The procedure is similar to the method of calculating median ranks.

Likelihood ratio bound The likelihood ratio bound also follows a simple methodology and are usually preferred over the Fisher's matrix bounds, specially when smaller sample sizes are analysed. They are based on a given likelihood equation and the results can be represented graphically as a contour plot. The extreme values of the contour plots are used for determining the required bounds.

Monte Carlo bound These could be parametric or nonparametric and is sometimes called the bootstrap method (parametric or non-parametric). For the parametric case, a model is fitted to the data, often by maximum likelihood, and samples of random numbers are drawn from this fitted model. A more rigorous method of calculating pivotal confidence bounds also exist.

For the present study, Monte Carlo parametric confidence intervals were chosen as they are relatively straightforward to determine and provide good approximations of the confidence interval estimate. The steps followed for calculating the confidence intervals for parameters of the Weibull distribution using the parametric bootstrap method, are listed as follows:

(1) Calculation of Weibull distribution parameters for the 100 simulated Weibull distributions incorporating the effect of measurement uncertainty, also shown in Fig. 4.

(2) Generation of 100 virtual fibre strength data sets for each synthetic Weibull distribution generated in Step 1, by randomly extracting 30 data points for each set, from the given distributions. This results in a total of $100 \times 100$ virtual data sets and simulates the effect of sampling.

(3) Estimation of best fit Weibull distribution parameters for all the 10,000 synthetic sample sets.

(4) Calculation of $2.5^{\text {th }}$ and $97.5^{\text {th }}$ percentile values of these distribution parameters. These values would represent the lower and upper bounds of the $95 \%$ confidence region.

A total of 10,000 different synthetic fibre strength data sets were randomly extracted following the steps mentioned above. Each data set comprised 30 fibre strength values covering the entire possible range of fibre strengths, within limits. Each synthetic data set was fitted to a 2-parameter Weibull distribution. The parameters for each distribution were determined using the maximum likelihood method. All simulated distributions are shown in Fig. 5, with the ones based on measurement uncertainty being in the central region and are surrounded by the ones based on sampling. The shape and scale parameters for all simulated distributions are tabulated together. To determine the $95 \%$ confidence intervals, the $2.5^{\text {th }}$ and $97.5^{\text {th }}$ percentile values were extracted for the shape and scale parameters. The associated confidence interval estimated from the simulated results is given in Table 5 . The width of estimated $95 \%$ confidence interval for shape parameter $m$ is 1.99 which is more than half of the best estimate value while the interval estimated for the scale parameter $\sigma_{0}$ is $0.97 \mathrm{GPa}$ which is about a quarter of the best estimate value. The simulated Weibull parameters are also represented with the help of histograms of the simulated shape and scale parameters from the tabulated data set, as shown in Fig. 6. The Weibull parameters can be reported in the form of best estimates of the parameter along with its standard deviation. The best estimate is represented by the mean of the data sets. The uncertainty in Weibull parameters is represented by the standard deviation of the data sets, given in Table 5 and also shown as follows:

$$
m=3.38 \text { with } u(m)=0.51
$$




$$
\sigma_{0}=4.17 \mathrm{GPa} \text { with } u\left(\sigma_{0}\right)=0.25 \mathrm{GPa}
$$

The expanded uncertainties can be calculated using Equation 6 using $t_{95}=1.96$ for $95 \%$ level of confidence which gives $U_{95}(m)=1.00$ and $U_{95}\left(\sigma_{0}\right)=0.49$. The shape and scale parameters in terms of their expanded uncertainties are given as follows:

$$
m=3.38 \pm 1.00 \text {, for a confidence level of } 95 \%
$$

$\sigma_{0}=4.17 \pm 0.49 \mathrm{GPa}$, for a confidence level of $95 \%$

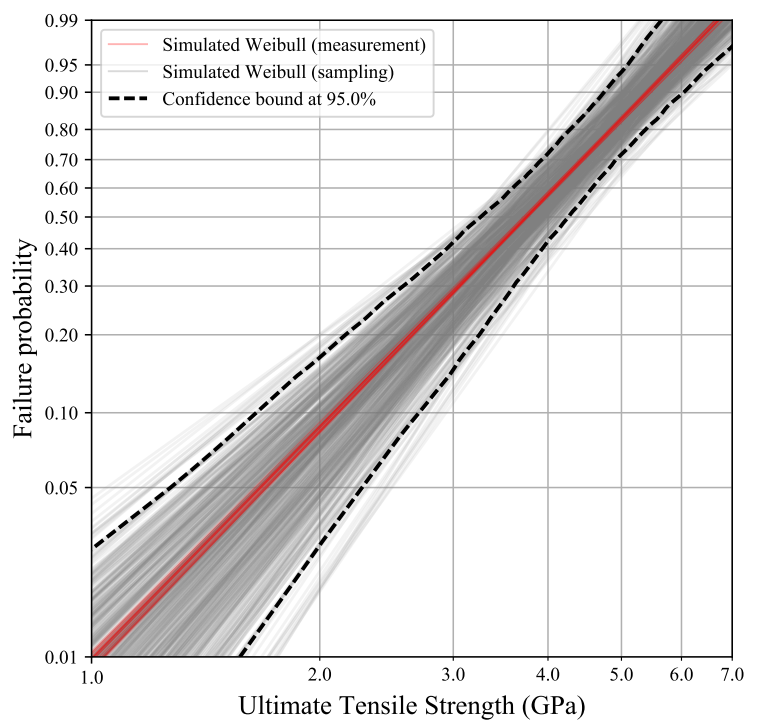

Fig. 5 Simulated Weibull distributions based on sampling and measurement uncertainty

It is observed that the uncertainty in Weibull parameters arising due to the effect of sampling is very large. These simulations were done using sample sizes of 30 fibres. It would therefore be recommended that to reduce the uncertainty due to the effect of sampling, a larger sample size is chosen for determining Weibull distribution parameters from single fibre test results. None of the works cited in Table 1 relating to single fibre tests (SFT) had made more than 30 tested fibres. Moreover, the studies with 30 tested fibres represent only one third of the SFT results mentioned.

\subsection{Confidence Region}

From the simulated Weibull distributions, a confidence region can also be determined. For this, it would be required to calculate a quantity called B-Strength, which is defined here.
Table 5 Weibull parameters and associated 95\% confidence interval for overall uncertainty due to measurement and sampling

\begin{tabular}{ccc}
\hline & $\begin{array}{c}\text { Shape } \\
\text { parameter } \\
m\end{array}$ & $\begin{array}{c}\text { Scale } \\
\text { parameter } \\
\sigma_{0}(G P a)\end{array}$ \\
\hline Mean & 3.38 & 4.17 \\
95\% Confidence Interval & $2.54-4.53$ & $3.69-4.66$ \\
Confidence width & 1.99 & 0.97 \\
Standard deviation & 0.51 & 0.25 \\
Expanded uncertainty at $95 \%$ & 1.00 & 0.49 \\
\hline
\end{tabular}

B-Strength: It may be of interest to determine the value at which a given percentage of the specimen population will fail. This quantity is similar to the one named B-life but since we are dealing with strengths in the present case, we can call it B-strength. Considering B50 strength for example, $50 \%$ of the fibre population is expected to have strength of less than this value. The scale parameter $\sigma_{0}$ then by definition is the B63.2 strength, i.e. $63.2 \%$ of the fibre population is expected to have a strength of less than this value. The B50 strength can be determined by substituting the value for $50 \%$ failure probability, i.e. 0.50 in the Weibull failure distribution equation given by Equation 1. A direct way to determine the B50 strength is by drawing a horizontal line at failure probability of 0.50 in the Weibull plot given in Fig. 3, finding its intersection with the best estimated Weibull line, and reading the corresponding strength value from the horizontal axis. The B50 strength is calculated to be $3.74 \mathrm{GPa}$ for the present case, which means that $50 \%$ of the fibres in the population are expected to have a strength of less than 3.74 GPa. Hence, B50 strength can also be called as median failure strength.

The steps for the calculation of a confidence region are as follows:

(1) From a simulated distribution, B-strength is calculated for all failure probabilities from 0.01 to 1.00, at intervals of 0.01 (i.e. B01, B02,..., B99, B100).

(2) Similarly, for all other simulated distributions, Bstrength values are calculated for all failure probabilities.

(3) For a given failure probability, all calculated Bstrength values coming from different simulated distributions are tabulated together. This is repeated for each failure probability.

(4) If a $95 \%$ confidence level is seek, the $2.5^{\text {th }}$ and $97.5^{\text {th }}$ percentile values for each B-strength are then con- 

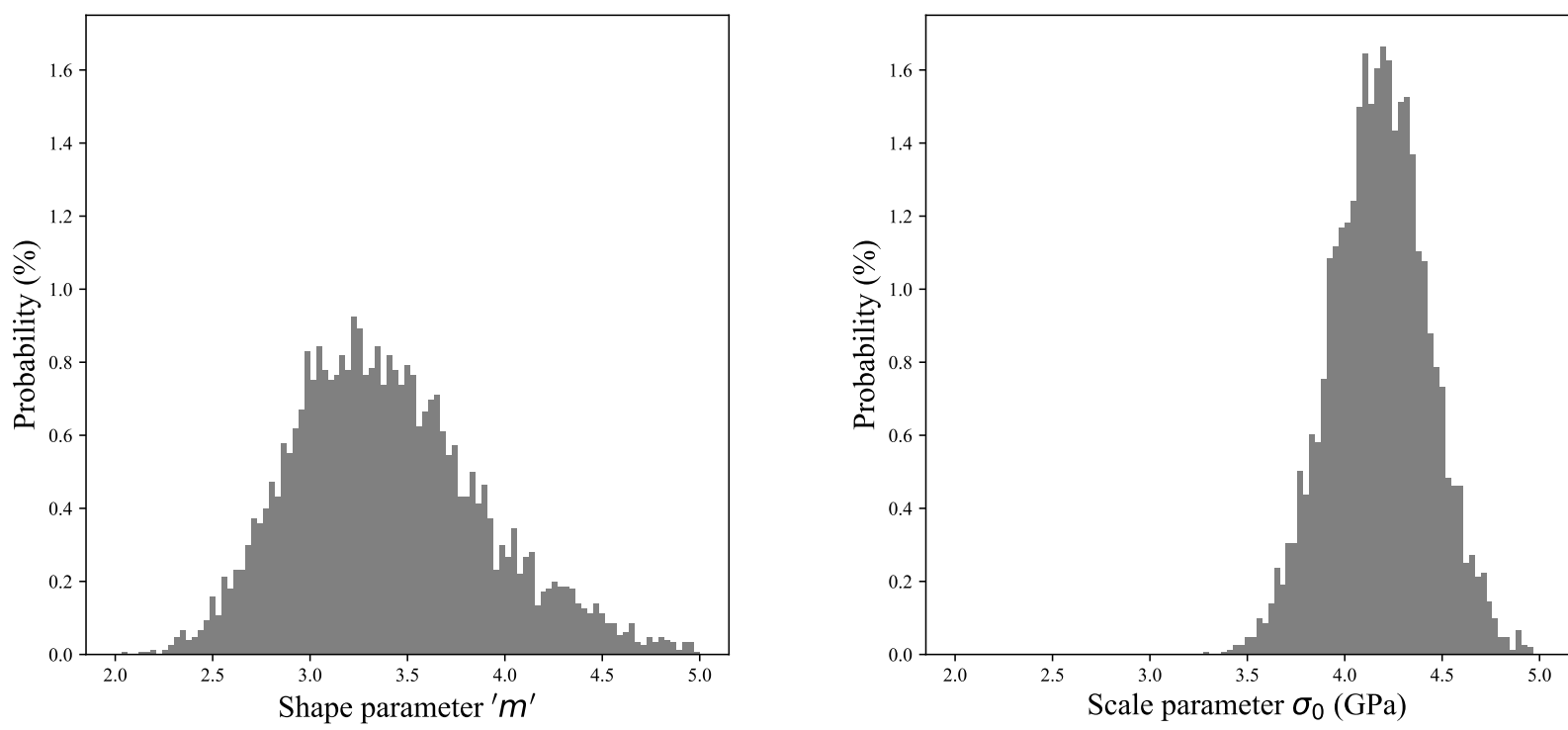

Fig. 6 Histogram of the observed parameter values of the simulated distributions for: a) Shape parameter b) Scale parameter

nected, to form the upper and lower bounds of the confidence region, respectively, as shown in Fig. 5.

(5) The region between these two confidence bounds gives the $95 \%$ confidence region.

For selective B-strengths, the best estimated values along with the $95 \%$ confidence intervals for uncertainty due to the combined effect of measurement uncertainty and sampling are shown in Table 6. For the B50 strength, for example, the best estimate is 3.74 $\mathrm{GPa}$. All best estimate values are the results coming from the Weibull distribution obtained from the experimental results. Considering the uncertainty, the $95 \%$ confidence interval in which the B50 strength is expected to lie is $3.25-4.24 \mathrm{GPa}$, i.e. within a bound width of 0.99 GPa. Similarly, other B-strength values can also be determined. It can be seen from Table 6 that width of the $95 \%$ confidence interval is fairly consistent for all B-strengths.

\subsection{Effect of sample size}

Different authors have chosen different sample sizes for single fibre testing, as shown in Table 1. The sample size often varies between 10-50 [42]. The choice of the number of test results introduces an uncertainty on the estimated Weibull parameters. It is evident that Weibull parameters determined from large sample sizes are more reliable than those obtained using a small sample size [43].
Table 6 Best estimated values for selective B-strengths along with the $95 \%$ confidence intervals for overall uncertainty due to measurement and sampling

\begin{tabular}{cccc}
\hline B-strength & $\begin{array}{c}\text { Best Estimate } \\
(\mathrm{GPa})\end{array}$ & $\begin{array}{c}95 \% \\
\text { Confidence Interval } \\
(\mathrm{GPa})\end{array}$ & $\begin{array}{c}\text { Width } \\
(\mathrm{GPa})\end{array}$ \\
\hline B20 & 2.64 & $2.19-3.16$ & 0.97 \\
B40 & 3.40 & $2.96-3.91$ & 0.95 \\
B50 & 3.74 & $3.25-4.24$ & 0.99 \\
B60 & 4.08 & $3.58-4.55$ & 0.97 \\
B80 & 4.85 & $4.30-5.30$ & 1.00 \\
\hline
\end{tabular}

To obtain an indication of the effect of sample size on the estimated confidence interval, more fibre strength experiments were conducted following the same methodology. Three sets of experimental results having different sample sizes were extracted to determine their scale parameters $\sigma_{0}$ (i.e. B63.2 strength), and also to estimate the corresponding $95 \%$ confidence interval for uncertainty due to sampling by simulating Weibull distributions using the method explained in section 3.2. The estimated confidence intervals are given in Table 7 . It can be seen that as the sample size increases, the confidence interval on the scale parameter becomes narrower. The narrower the confidence interval, the less the uncertainty. Narrow confidence intervals thus represent more accurately estimated results. It would therefore be recommended that a sufficiently large set of exper- 
imentally generated fibre strength data is used for determining the representative Weibull distribution. This would minimize the uncertainty due to sampling randomness, and maximize the accuracy of the estimated distribution parameters.

Table 7 Effect of sample size on estimated $95 \%$ confidence interval of Weibull scale parameter $\sigma_{0}$ (B63.2 life)

\section{$95 \%$}

\begin{tabular}{cccc}
$\begin{array}{c}\text { Sample size } \\
\text { N }\end{array}$ & $\begin{array}{c}\text { Best Estimate } \\
(\mathrm{GPa})\end{array}$ & $\begin{array}{c}95 \% \\
\text { Confidence Interval } \\
(\mathrm{GPa})\end{array}$ & $\begin{array}{c}\text { Width } \\
(\mathrm{GPa})\end{array}$ \\
\hline 15 & 4.22 & $3.55-5.24$ & 1.49 \\
30 & 4.25 & $3.77-4.78$ & 1.01 \\
45 & 4.18 & $3.81-4.57$ & 0.76 \\
\hline
\end{tabular}

\section{Conclusions}

The influence of practical limitations on the accuracy of fibre strength distribution parameters has been studied in this article. Limitations such as unavoidable measurement uncertainties and the randomness introduced due to the sampling effect have been statistically analysed with the help of Monte Carlo bootstrap methods. Any measurement of fibre strength has some level of uncertainty associated with it, mainly due to limitations of the measuring equipment and the experimental processes used. It has been shown that when such a set of experimentally generated fibre strength data is represented with the help of a statistical function, these errors in individual fibre strength values are also propagated into the resulting statistical distribution, and thus add uncertainty to the distribution parameters obtained. The sampling randomness too has been shown to contribute to the uncertainty of these distribution parameters. The representativeness of the sample size chosen for analysis is thus very crucial. The uncertainties arising due to these two reasons, i.e. due to measurement uncertainty and due to sampling randomness have been quantified in terms of a range of possible values which the distribution parameters are expected to take. This confidence interval quantifies the expected variability in distribution parameters arising due to overall effect of the measurement inaccuracies and sampling randomness.

It has been shown that although both the causes of uncertainty are critical, the sampling randomness has a larger influence on the uncertainty of the estimated fibre strength results while the uncertainty resulting due to the uncertain measurement of individual fibre strength was not very substantial. However, this would be true only for fibre strength data generated using a sufficiently long gauge length. This is because the effect of measurement limitations is small for tests conducted at large gauge lengths and the measured tensile strength is more accurate. However, for shorter gauge lengths, measurement uncertainty of the instruments and experimental constraints limits the accuracy of the fibre strength data generated. Their effect on the uncertainty of the distribution parameters in this case is also expected to be significant [15].

For minimizing the uncertainty due to sampling randomness, it has been recommended that a sufficiently large set of experimentally generated fibre strength data is used, in order to minimize the sampling effect and maximize the accuracy of the estimated distribution parameters. It has also been demonstrated with the help of experimental results that the larger is the size of the fibre strength data set used for analysis, the greater is the accuracy of the determined results.

This knowledge about the uncertainty in fibre strength distribution parameters would empower users to estimate the confidence that can be placed on the predictions made by composite strength models on the damage and failure behaviour of composite structures. This can be done by using the obtained fibre strength properties (along with uncertainty) as input for the predictive models to determine the variability in the model predictions. The knowledge about the contributions of different factors which lead to uncertainty in fibre strength results would also allow researchers to take appropriate measures for minimising these uncertainties. Using the results from this study, the contribution of the critical parameters which cause uncertainty in measurement of fibre tensile strength has been determined in another study [15]. More accurate fibre strength input data would thus enhance the capability of composite strength models to estimate the behaviour of composite structures more accurately.

Acknowledgements The research leading to these results has been done within the framework of the FiBreMoD project and has received funding from the European Union's Horizon 2020 research and innovation programme under the Marie Sklodowska-Curie grant agreement No 722626.

\section{References}

1. S. Joannès, F. Islam, L. Laiarinandrasana, Uncertainty in Fibre Strength Characterisation Due to Uncertainty in Measurement and Sampling Randomness, Applied Composite Materials 27 (3) (2020) 165-184. doi:10.1007/ s10443-020-09803-9. 
2. X. Zhang, J. Zhao, Z. Wang, Burst pressure prediction and structure reliability analysis of composite overwrapped cylinder, Applied Composite Materials 25 (6) (2018) 1269-1285. doi:10.1007/s10443-017-9665-x. URL https://doi.org/10.1007/s10443-017-9665-x

3. H. Chou, A. Bunsell, G. Mair, A. Thionnet, Effect of the loading rate on ultimate strength of composites. application: Pressure vessel slow burst test, Composite Structures 104 (2013) 144 - 153. doi:https://doi.org/10. 1016/j. compstruct. 2013.04.003.

4. Overview of the global composites market - at the crossroads, JEC Group, 2017.

5. A. R. Bunsell, A. Thionnet, H. Y. Chou, Intrinsic safety factors for glass \& carbon fibre composite filament wound structures, Applied Composite Materials 21 (1) (2014) 107-121. doi:10.1007/s10443-013-9367-y. URL https://doi.org/10.1007/s10443-013-9367-y

6. P. Ilankeeran, P. Mohite, S. Kamle, Axial tensile testing of single fibres, Modern Mechanical Engineering (4) (2012) 151-156. doi:10.4236/mme.2012.24020.

7. A. Thionnet, H.-Y. Chou, A. Bunsell, Fibre break failure processes in unidirectional composites. part 1: Failure and critical damage state induced by increasing tensile loading, Applied Composite Materials 22 (2) (2015) 119-140. doi:10.1007/s10443-014-9397-0. URL https://doi.org/10.1007/s10443-014-9397-0

8. E. K. Gamstedt, S. Östlund, Fatigue propagation of fibrebridged cracks in unidirectional polymer-matrix composites, Applied Composite Materials 8 (6) (2001) 385-410. doi:10.1023/A:1012677604599.

URL https://doi.org/10.1023/A:1012677604599

9. A. Kaddour, M. Hinton, Maturity of 3d failure criteria for fibre-reinforced composites: Comparison between theories and experiments: Part b of wwfe-ii, Journal of Composite Materials 47 (6-7) (2013) 925-966. arXiv: https://doi.org/10.1177/0021998313478710, doi:10. 1177/0021998313478710.

URL https://doi.org/10.1177/0021998313478710

10. A. Bunsell, L. Gorbatikh, H. Morton, S. Pimenta, I. Sinclair, M. Spearing, Y. Swolfs, A. Thionnet, Benchmarking of strength models for unidirectional composites under longitudinal tension, Composites Part A: Applied Science and Manufacturing 111 (2018) 138 - 150. doi:https: //doi.org/10.1016/j. compositesa.2018.03.016. URL http://www.sciencedirect.com/science/ article/pii/S1359835X18301180

11. H. Y. Chou, A. Thionnet, A. Mouritz, A. R. Bunsell, Stochastic factors controlling the failure of carbon/epoxy composites, Journal of Materials Science 51 (1) (2016) 311-333. doi:10.1007/s10853-015-9390-5.

URL https://doi.org/10.1007/s10853-015-9390-5

12. Y. Swolfs, I. Verpoest, L. Gorbatikh, A review of input data and modelling assumptions in longitudinal strength models for unidirectional fibre-reinforced composites, Composite Structures 150 (2016) 153 - 172. doi: https://doi.org/10.1016/j. compstruct.2016.05.002.

13. F. Islam, S. Joannès, S. Bucknell, Y. Leray, A. Bunsell, L. Laiarinandrasana, Improvements in determination of carbon fibre strength distribution using automation and statistical data analysis, in: Proceedings of the Fiber Society's Spring 2018 Conference, Tokyo, Japan, 2018, June $12-14$.

14. JCGM 100: Evaluation of measurement data - Guide to the expression of uncertainty in measurement, 2010.

15. F. Islam, S. Joannès, L. Laiarinandrasana, Evaluation of critical parameters in tensile strength measurement of single fibres, Journal of Composites Science 3 (3) (2019). doi: $10.3390 / j \operatorname{cs} 3030069$.

URL https://www .mdpi.com/2504-477X/3/3/69

16. F. Islam, S. Joannès, S. Bucknell, Y. Leray, A. Bunsell, L. Laiarinandrasana, Towards accurate and efficient single fibre characterization to better assess failure strength distribution, in: Proceedings of the 18th European Conference on Composite Materials (ECCM-2018), Greece, Athens, 2018, June 25-28.

17. W. Weibull, A statistical distribution function of wide applicability, Journal of Applied Mechanics (1951) 293297.

18. W. J. Padgett, S. D. Durham, A. M. Mason, Weibull analysis of the strength of carbon fibers using linear and power law models for the length effect, Journal of Composite Materials 29 (14) (1995) 1873-1884. doi: 10.1177/002199839502901405.

19. M.-H. Berger, D. Jeulin, Statistical analysis of the failure stresses of ceramic fibres: Dependence of the Weibull parameters on the gauge length, diameter variation and fluctuation of defect density, Journal of Materials Science 38 (13) (2003) 2913-2923. doi:10.1023/A: 1024405123420.

20. W. Yu, J. Yao, Tensile strength and its variation of PANbased carbon fibers. I. Statistical distribution and volume dependence, Journal of Applied Polymer Science 101 (5) 3175-3182. arXiv:https://onlinelibrary.wiley.com/ doi/pdf/10.1002/app. 23399, doi:10.1002/app. 23399.

21. B. Yavin, H. E. Gallis, J. Scherf, A. Eitan, H. D. Wagner, Continuous monitoring of the fragmentation phenomenon in single fiber composite materials, Polymer Composites 12 (6) 436-446. doi:10.1002/pc.750120609.

22. Z. Chi, T. W. Chou, G. Shen, Determination of single fibre strength distribution from fibre bundle testings, Journal of Materials Science 19 (10) (1984) 3319-3324. doi: $10.1007 /$ BF00549820.

23. J. Andersons, R. Joffe, M. Hojo, S. Ochiai, Glass fibre strength distribution determined by common experimental methods, Composites Science and Technology 62 (1) (2002) 131 -145. doi:https://doi.org/10.1016/ S0266-3538(01) 00182-8.

24. S. Feih, K. Manatpon, Z. Mathys, A. G. Gibson, A. P. Mouritz, Strength degradation of glass fibers at high temperatures, Journal of Materials Science 44 (2) (2009) 392400. doi:10.1007/s10853-008-3140-x.

25. H. Xiao, Y. Lu, M. Wang, X. Qin, W. Zhao, J. Luan, Effect of gamma-irradiation on the mechanical properties of polyacrylonitrile-based carbon fiber, Carbon 52 (2013) 427 - 439. doi:https://doi.org/10.1016/j.carbon. 2012.09 .054 .

26. S. Deng, L. Ye, Y.-W. Mai, H.-Y. Liu, Evaluation of fibre tensile strength and fibre/matrix adhesion using single fibre fragmentation tests, Composites Part A: Applied Science and Manufacturing 29 (4) (1998) 423-434. doi: https://doi.org/10.1016/S1359-835X (97) 00094-8.

27. V. Lutz, J. Duchet-Rumeau, N. Godin, F. Smail, F. Lortie, J. Gérard, Ex-PAN carbon fibers vs carbon nanotubes fibers: From conventional epoxy based composites to multiscale composites, European Polymer Journal 106 (2018) 9 -18. doi:https://doi.org/10.1016/ j.eurpolymj.2018.06.023.

28. W. Na, G. Lee, M. Sung, H. N. Han, W.-R. Yu, Prediction of the tensile strength of unidirectional carbon fiber composites considering the interfacial shear strength, Composite Structures 168 (2017) 92 - 103. doi:https: //doi.org/10.1016/j. compstruct.2017.02.060.

29. M. Matveev, A. Long, I. Jones, Modelling of textile composites with fibre strength variability, Composites Sci- 
ence and Technology 105 (2014) 44 - 50. doi:https: //doi.org/10.1016/j.compscitech.2014.09.012.

30. T. Zhang, L. Qi, S. Li, X. Chao, W. Tian, J. Zhou, Evaluation of the effect of PyC coating thickness on the mechanical properties of T700 carbon fiber tows, Applied Surface Science 463 (2019) 310 - 321. doi:https: //doi.org/10.1016/j.apsusc. 2018.08.195.

31. Y. Zhou, Y. Wang, Y. Xia, S. Jeelani, Tensile behavior of carbon fiber bundles at different strain rates, Materials Letters 64 (3) (2010) 246 - 248. doi:https://doi.org/ $10.1016 / j$.matlet.2009.10.045.

32. Standard test method for tensile strength and Young's modulus of fibers, ASTM Standards ASTM C1557 - 14 (2014).

33. A. R. Bunsell, J. W. S. Hearle, A mechanism of fatigue failure in nylon fibres, Journal of Materials Science 6 (10) (1971) 1303-1311. doi:10.1007/BF00552044.

34. A. Marcellan, A. Bunsell, L. Laiarinandrasana, R. Piques, A multi-scale analysis of the microstructure and the tensile mechanical behaviour of polyamide 66 fibre, Polymer 47 (1) (2006) 367 - 378. doi:https://doi.org/10.1016/ j. polymer.2005.10.093.

35. J. Wollbrett-Blitz, S. Joannès, R. Bruant, C. Le Clerc, M. Romero De La Osa, A. Bunsell, A. Marcellan, Multiaxial mechanical behavior of aramid fibers and identification of skin/core structure from single fiber transverse compression testing, Journal of Polymer Science Part B: Polymer Physics 54 (3) (2016) 374-384. doi: 10.1002/polb. 23763.

36. V. Jauzein, A. Bunsell, Bio-composite aspects of silk: the sericin sheath acting as a matrix, Journal of Materials Science 47 (7) (2012) 3082-3088. doi:10.1007/ s10853-011-6141-0.

URL https://doi.org/10.1007/s10853-011-6141-0

37. J. M. H. Ramirez, A. R. Bunsell, P. Colomban, Microstructural mechanisms governing the fatigue failure of polyamide 66 fibres, Journal of Materials Science 41 (22) (2006) 7261-7271. doi:10.1007/s10853-006-0421-0. URL https://doi.org/10.1007/s10853-006-0421-0

38. C. Le Clerc, B. Monasse, A. R. Bunsell, Influence of temperature on fracture initiation in pet and pa66 fibres under cyclic loading, Journal of Materials Science 42 (22) (2007) 9276-9283. doi:10.1007/s10853-007-1864-7. URL https://doi.org/10.1007/s10853-007-1864-7

39. J. VanderPlas, Frequentism and Bayesianism: a Pythondriven primer, in: Proceedings of the 13th Python in Science conference (SCIPY 2014), 2014.

40. J. Symynck, F. D. Bal, Monte-carlo pivotal confidence bounds for Weibull analysis, with implementations in $\mathrm{R}$, in: Proceedings of the XVIth International Scientific Conference "Tehnomus", "Stefan cel Mare" University of Suceava - ROMANIA, 2011.

41. R. B. Abernethy, The New Weibull Handbook, 2008.

42. P. Zinck, E. Mäder, J. F. Gerard, Role of silane coupling agent and polymeric film former for tailoring glass fiber sizings from tensile strength measurements, Journal of Materials Science 36 (21) (2001) 5245-5252. doi: 10.1023/A: 1012410315601 .

43. J. Thomason, On the application of Weibull analysis to experimentally determined single fibre strength distributions, Composites Science and Technology 77 (2013) 74 - 80. doi:https://doi.org/10.1016/j.compscitech. 2013.01 .009 . 